Forum 2019 · 34:230

https://doi.org/10.1007/s12312-019-0608-3

Online publiziert: 23. April 2019

(c) Springer Medizin Verlag $\mathrm{GmbH}$, ein Teil von Springer Nature 2019

\title{
Deutsche Krebsgesellschaft startet Pilotprojekt zur ärztlichen Zweitmeinung
}

für eine Zertifizierung erfüllt sein müssen, leiten sich aus den Empfehlungen der aktuell geltenden onkologischen Leitlinien ab. Auf diese Weise ist sichergestellt, dass das aktuell verfügbare medizinische Wissen in die Therapieentscheidung einfließt.

Insgesamt mehr als 1000 Zentren in über 440 Krankenhäusern weisen mittlerweile ein Zertifikat der DKG auf, rund $40 \%$ aller Patientinnen und Patienten mit der Erstdiagnose Krebs in Deutschland werden an einem DKG-zertifizierten Zentrum behandelt. „Verschiedene Studien zeigen, dass statistisch gesehen das Gesamtüberleben der Patienten an zertifizierten Darmkrebszentren deutlich höher ist als an nicht zertifizierten Zentren", erklärte Prof. Dr. Thomas Seufferlein, Vizepräsident der DKG anlässlich eines Pressegesprächs in Berlin. Umso wichtiger sei es, dass diejenigen, die außerhalb von zertifizierten Zentren behandelt werden, die Möglichkeit erhielten, eine qualifizierte Zweitmeinung durch ein interdisziplinär besetztes Tumorboard einzuholen.

Doch auch bei Patienten mit einer Therapieempfehlung eines zertifizierten Zentrums kann der Wunsch nach zusätzlicher Abklärung bestehen. „Das gilt vor allem bei komplexen Krankheitsbildern, oder wenn, wie etwa in den frühen Sta- dien eines Prostatakarzinoms, verschiedene Therapieoptionen möglich sind und die Entscheidung schwer fällt", so Prof. Dr. Thorsten Schlomm, Urologe und Leiter des DKG-zertifizierten Prostatakarzinomzentrums der Charité Universitätsmedizin Berlin. „Wir freuen uns, dass Patientinnen und Patienten künftig auf die zertifizierten Zentren als qualifizierte Zweitmeinungsgeber zurückgreifen können und hoffen dabei sehr auf eine breite Unterstützung durch die Krankenkassen." Auch wenn einige Kassen derzeit eine ärztliche Zweitmeinung bei Krebspatienten erstatten, eine gesetzliche Verpflichtung für eine Kostenübernahme besteht bei onkologischen Indikationen nicht. Außerdem ist bei manchen der bestehenden Zweitmeinungsangebote derzeit unklar, ob die Begutachtung durch eine Tumorkonferenz erfolgt und die aktuell geltenden Leitlinien sowie die Patientenperspektive ausreichend berücksichtigt werden.

\section{Pressekontakt}

\section{Dr. Katrin Mugele}

Pressestelle der Deutschen Krebsgesellschaft e.V.

Tel: 030 3229329-60

E-Mail:mugele@krebsgesellschaft.de 